Boise State University

ScholarWorks

Physics Faculty Publications and Presentations

Department of Physics

$2-2021$

Interaction of Alpha-Crystallin with Phospholipid Membranes

Laxman Mainali

Boise State University

William J. O'Brien

Medical College of Wisconsin

Raju Timsina

Boise State University 
This is an author-produced, peer-reviewed version of this article. The final, definitive version of this document can be found online at Current Eye Research, published by Taylor \& Francis. Copyright restrictions may apply. doi: 10.1080/02713683.2020.1786131

\title{
Interaction of Alpha-Crystallin with Phospholipid Membranes
}

\author{
Laxman Mainali \\ Department of Physics \\ Boise State University \\ Boise, Idaho, USA \\ laxmanmainali@boisestate.edu \\ and \\ Department of Biophysics \\ Medical College of Wisconsin \\ Milwaukee, Wisconsin, USA
}

\author{
William J. O'Brien \\ Department of Ophthalmology and Visual Sciences \\ Medical College of Wisconsin \\ Milwaukee, Wisconsin, USA \\ and
}

Raju Timsina

Department of Physics

Boise State University

Boise, Idaho, USA

\begin{abstract}
Purpose/Aim: The amount of membrane-bound $\alpha$-crystallin increases significantly with age and cataract formation, accompanied by a corresponding decline in the level of $\alpha$-crystallin in the lens cytoplasm. The purpose of this research is to evaluate the binding affinity of $\alpha$-crystallin to the phospholipid membranes as well as the physical properties of the membranes after $\alpha$ crystallin binding.
\end{abstract}

Materials and Methods: The continuous wave and saturation recovery electron paramagnetic resonance (EPR) methods were used to obtain the information about the binding affinity and the physical properties of the membrane. In this approach, the cholesterol analogue spin label CSL was incorporated in the membrane and the binding of $\alpha$-crystallin to the membrane was monitored by this spin label. Small uni-lamellar vesicles were prepared from 1-palmitoyl-2oleoylphosphatidylcholine (POPC) with 1\% of CSL. The measured membrane properties included the mobility parameter, fluidity and the oxygen transport parameter.

Results: The binding affinity $\left(K_{a}\right)$ of $\alpha$-crystallin with the POPC membrane was estimated to be $4.9 \pm 2.4 \mu \mathrm{M}^{-1}$. The profiles of mobility parameter showed that mobility parameter decreased with an increase in the binding of $\alpha$-crystallin. The profiles of spin-lattice relaxation rate showed that the spin-lattice relaxation rate decreased with an increase in binding. These results show that the binding of $\alpha$-crystallin makes the membrane more immobilized near the head group region of the phospholipids. Furthermore, the profiles of the oxygen transport parameter indicated that the oxygen transport parameter decreased with an increase of binding, indicating the binding of $\alpha$-crystallin forms a barrier for the passage of non-polar molecules which supports the barrier hypothesis.

Conclusions: The binding of $\alpha$-crystallin to the membrane alters the physical properties of the membranes, and this plays a significant role in modulating the integrity of the membranes. EPR techniques are useful in studying $\alpha$-crystallin membrane interactions.

Keywords: $\alpha$-crystallin, phospholipid membrane, EPR, binding, fluidity

Word count: 6072

\section{Introduction}

Epidemiological studies have shown that cataract development is a multifactorial process involving genetic and environmental factors $\underline{1,2}$. It has been reported that ionizing radiation (IR) exposure is an important environmental factor in cataract development $\stackrel{3}{-}$. IR generates free radical and induces oxidative stress that damages lens protein resulting in protein aggregation, precipitation, and loss of lens transparency ${ }^{3}$. Oxidation of lipids in the membrane via oxidative stress leads to the disruptions of membrane structure ${ }^{3}$. In addition to genetics, factors that can cause cataract formation are age $\frac{4,5}{,}$, vitrectomy $\underline{6,7}$, and hyperbaric oxygen treatment $\frac{8-10}{10}$. It is widely postulated that cataract formation results from any type of oxidative stress that perturbs the structure of lens fiber-cell membranes, disrupts the function 
This is an author-produced, peer-reviewed version of this article. The final, definitive version of this document can be found online at Current Eye Research, published by Taylor \& Francis. Copyright restrictions may apply. doi: 10.1080/02713683.2020.1786131

of intrinsic proteins, and promotes the aggregation of cytosolic proteins, crystallins $4,10,11$. Aggregation of crystallins is the significant factor in cataract formation $\underline{12, \underline{13}}$. It is believed that the associations between fiber-cell plasma membrane and crystallins are an important phenomenon in the aging lens by which crystallin insolubilization occurs $\stackrel{14-16}{ }$. It has been reported that all water insoluble crystallins in aged human lenses are membrane bound $\frac{17}{}$.

The three classes of crystallins are $\alpha-, \beta-$, and $\gamma$, which account for nearly $90 \%$ of the lens proteins $\frac{18-20}{}$. Among them, $\alpha$-crystallin comprises up to $40 \%$ of total lens proteins $\stackrel{18,21}{2}$ and has the strongest affinity toward lens membranes $\underline{22-27}$. Several reports indicate that levels of $\alpha$-crystallin in the lens cytoplasm decline with age and cataract progression, and are accompanied by a corresponding increase in the amount of membrane-bound $\alpha$-crystallin $15,28, \underline{29}$. A recent clinical study showed that the level of the unbound form of $\alpha$-crystallin decreased with cataract progression $\underline{29}$. The association of $\alpha$-crystallin with the membrane is accompanied by increased light scattering. It was shown that during aging, and in the case of cataract formation, the association of $\alpha$-crystallin with membrane and light scattering increases $22,30,31$. It has also been hypothesized that the binding of $\alpha$-crystallin to the fiber cell plasma membrane contributes to the development of nuclear cataracts by occluding membrane pores and forming a barrier to diffusion $5,14,32$.

Several studies have been performed to investigate the binding of $\alpha$-crystallin to lens membrane $22-28,33-38$. It has also been proposed earlier that lipid moiety alone of the lens membrane may be responsible for $\alpha$-crystallin membrane binding $34, \underline{36}, \underline{38}, \underline{39}$. However, the mechanism by which $\alpha$-crystallin associates with membrane's lipid is unclear. Here we have developed the Electron Paramagnetic Resonance (EPR) approach to investigate the binding of $\alpha$-crystallin to pure phospholipid membrane (protein free membrane). The EPR approach allows us to estimate the binding affinity $\left(\mathrm{K}_{\mathrm{a}}\right)$ as well as the physical properties of membrane after the binding of $\alpha$-crystallin . The physical properties of membrane studied here include mobility parameter, fluidity parameter and the oxygen transport parameter (OTP). The EPR approach uses cholesterol analog spin label (CSL) near the membrane surface which gives us the unique opportunity to obtain the information about the membrane surface occupied by $\alpha$-crystallin. Here the lipid membrane chosen for the study is phosphatidylcholine (PC) membrane because PC is the most abundant phospholipid (PL) in eye lenses of short-life-span animals ${ }^{40}$.

\section{Materials and Methods}

\subsection{Materials}

One-palmitoy-2-oleoyl-sn-glycero-3-phosphatidylcholine (POPC) was purchased from Avanti Polar Lipids, Inc. (Alabaster, AL, USA). Cholesterol analog cholestane spin label (CSL) was obtained from Molecular Probes (Eugene, OR, USA). We have chosen CSL spin-label in the proposed research because the nitroxide moiety of CSL is close to the membrane surface and it was reported earlier by another research group $\underline{34}$ that the headgroup region of the membrane becomes less mobile with binding of $\alpha$-crystallin. Bovine eye lens $\alpha$-crystallin and other chemicals (of at least reagent grade) were purchased from Sigma-Aldrich (St. Louis, MO, USA). The $\alpha$-crystallin (C4163) purchased from Sigma-Aldrich was used without any further purification. The molecular weight of $\alpha$-crystallin was estimated to be $20.35 \mathrm{kDa}$ based on the information provided by Sigma-Aldrich via phone (i.e, $\alpha \mathrm{A}=19.8 \mathrm{kDa}, \alpha \mathrm{B}=22 \mathrm{kDa}, \alpha \mathrm{A}: \alpha \mathrm{B}$ $=3: 1$ ). As estimated by other research groups $\frac{41,42}{}$, the isolation of $\alpha$-crystallin from native bovine eye lens consists of about $6 \%$ impurities. The possibility of the binding of impurities to the membrane cannot be discarded; however, with $\alpha$-crystallin being the most abundant fraction, the change in the lipid organization and dynamics sensed by CSL in the membrane is mostly due to $\alpha$-crystallin. The possible effect of impurities on $K_{a}$ and membrane properties can be eliminated by using recombinant pure $\alpha$-crystallin. We plan to use recombinant pure $\alpha$-crystallin in our future studies, as used by other research groups $\underline{35,43}$.

\subsection{Preparation of Small Unilamellar Vesicles (SUVs)}

The small unilamellar vesicles (SUVs) were prepared by sonication of the dispersion of large multilamellar vesicle (LMV) suspensions $\stackrel{44}{ }$ made of POPC containing $1 \mathrm{~mol} \%$ of CSL. Chloroform solutions of POPC and CSL were mixed to attain the desired spin-label concentration. A chloroform solution with a final volume of $75 \mu \mathrm{L}$ was added to the test tube of $1.2 \mathrm{~mL}$ of Hepes buffer ( $10 \mathrm{mM}$ Hepes, $100 \mathrm{mM} \mathrm{NaCl}, \mathrm{pH}=7.4)$ at room temperature. LMVs were

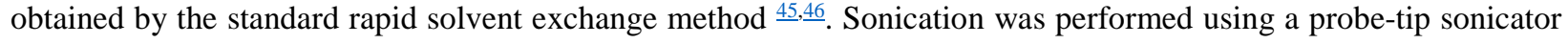
(Fisher Scientific, Model 550). Five to seven $15 \mathrm{~s}$ sonication cycles followed by $15 \mathrm{~s}$ cooling in ice were sufficient to transfer the milky suspension of LMVs into a slightly hazy transparent solution. Sonication produces SUVs approximately $30 \mathrm{~nm}$ in diameter ${ }^{44}$. The final concentration of POPC was $25 \mathrm{mg} / \mathrm{mL}$. $3.54 \mathrm{mg} / \mathrm{mL}$ stock of $\alpha$-crystallin was prepared in a Hepes buffer. 
This is an author-produced, peer-reviewed version of this article. The final, definitive version of this document can be found online at Current Eye Research, published by Taylor \& Francis. Copyright restrictions may apply. doi: 10.1080/02713683.2020.1786131

\section{3. a-Crystallin Membrane Interaction}

The continuous wave (CW) EPR and saturation recovery (SR) EPR methods are powerful tools that can provide a great deal of information on the binding of $\alpha$-crystallin with membrane. In this approach, the cholesterol analogue CSL is incorporated in the membrane and the binding of $\alpha$-crystallin to the membrane is monitored by this spin label. Fig. 1 is a schematic drawing of an $\alpha$-crystallin bound membrane along with a CSL. Here, the membrane is the SUVs with only the outer membrane surface exposed to $\alpha$-crystallin. The cholesterol analogue spin label (CSL, see Fig. 1 for its location in the membrane) is incorporated in the membrane, and the EPR signal coming from CSL provides information about the percentage of the membrane surface occupied by $\alpha$-crystallin. The EPR signal from CSL also gives the information about the change in the physical properties (mobility, fluidity and the oxygen transport parameter) of membrane after the binding of $\alpha$-crystallin.

\subsection{Preparation of Samples for EPR Measurements}

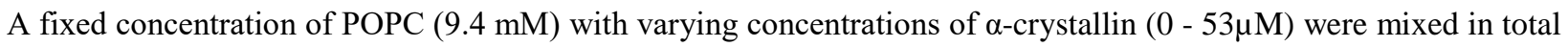
volume of $70 \mu \mathrm{L}$ and incubated for $16 \mathrm{~h}$ at $37^{\circ} \mathrm{C}$ with gentle shaking in a Corning LSE benchtop shaking incubator (Corning. NY, USA). The sample was then transferred to a $0.6 \mathrm{~mm}$ i.d. capillary made of gas-permeable methylpentene polymer (TPX) ${ }^{47}$, which was used for CW and SR EPR measurements. To measure time course of binding, both the concentration of POPC $(9.4 \mathrm{mM})$ and $\alpha$-crystallin $(35 \mu \mathrm{M})$ were fixed and incubated with gentle shaking at $37^{\circ} \mathrm{C}$ with varying times. The samples at different time intervals were then transferred to TPX capillary for CW EPR measurements. All CW and SR EPR measurements were performed at $37^{\circ} \mathrm{C}$.

\subsection{EPR Measurements}

CW EPR spectra were recorded with a Bruker EMX spectrometer equipped with temperature-control accessories. All spectra were obtained with modulation amplitude of $1.0 \mathrm{G}$ and incident microwave power of $5.0 \mathrm{~mW}$. Samples were thoroughly deoxygenated, yielding correct EPR lineshapes.

SR measurements were performed at X-band on a home-built spectrometer with major hardware improvements and equipped with a loop-gap resonator (LGR) ${ }^{48}$. One of the improvements was that the pump arm power amplifier was replaced with a new power amplifier to provide more saturating pump power to the sample. With this upgrade to the equipment, the pump arm in the SR system is capable of delivering a pulse width as narrow as $10 \mathrm{~ns}$ at a $1 \mathrm{~W}$ power

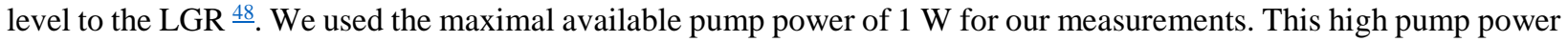
is especially significant when the experiment is done with the samples equilibrated with air ( $30 \%$ and $50 \%$ air in the current project). Equilibration with air shortened the spin-lattice relaxation time ( $\left.T_{1}\right)$ value, and the availability of this level of pump power ensures saturation of the sample with the narrow pump pulse widths needed to measure the shorter $T_{1} \mathrm{~S}$ values accurately. $T_{1} \mathrm{~S}$ of spin label were determined by analyzing the SR signal of the central line obtained by short-pulse SR EPR at X-band $49, \underline{50}$. The concentration of oxygen in the sample was controlled by equilibration with the same gas that was used for the temperature control (i.e., a controlled mixture of nitrogen and dry air adjusted with flow meters [Matheson Gas Products, model 7631H-604] ) ${ }^{47}$. Typically, $10^{5}-10^{6}$ decays were acquired with 2048 data points on each decay with sampling interval 5 ns or 10 ns. The total accumulation time was typically $2-5$ min. SR signals were fitted by single- and double exponential functions. Single exponentials fits were sufficient.

\subsection{Theory Outline for Evaluation of OTP}

The OTP, $W(\mathrm{x})$, was introduced as a convenient quantitative measure of the rate of the collision between the spin label and molecular oxygen by Kusumi et al. $\frac{51}{}$ as

$W(\mathrm{x})=T_{1}^{-1}($ Air, $\mathrm{x})-T_{1}^{-1}\left(\mathrm{~N}_{2}, \mathrm{x}\right)$

where the $T_{1} \mathrm{~S}$ are the spin-lattice relaxation times of the nitroxides in samples equilibrated with atmospheric air and nitrogen, respectively. The collision rate is also proportional to the local oxygen concentration $C(\mathrm{x})$ and the local oxygen-diffusion coefficient $D(\mathrm{x})$ at a "depth" $\mathrm{x}$ in the membrane that is in equilibrium with atmospheric air:

$W(\mathrm{x})=A D(\mathrm{x}) C(\mathrm{x}), A=8 \pi p r_{\mathrm{o}}$ 
This is an author-produced, peer-reviewed version of this article. The final, definitive version of this document can be found online at Current Eye Research, published by Taylor \& Francis. Copyright restrictions may apply. doi: 10.1080/02713683.2020.1786131

where $r_{\mathrm{o}}$ is the interaction distance between oxygen and the nitroxide moiety of the spin label (4.5 $\AA$ ) $\underline{52}$ and $p$ is the probability that an observable event occurs when a collision occurs ( $A$ is remarkably independent of the hydrophobicity and viscosity of the solvent and of spin label species $\frac{53-55}{}$ ). Kusumi et al. $\frac{51}{1}$ concluded that the OTP is a useful monitor of membrane fluidity that reports on translational diffusion of small molecules. To obtain the OTP, in principle, two SR measurements could be performed for the sample equilibrated with nitrogen and air (see Eq. [1]). However, the $T_{1}$ for samples equilibrated with air is too short to be measured with great accuracy. Thus, SR measurements were carried out as a function of oxygen concentration i.e. percent air up to $50 \%$ air and extrapolation to $100 \%$ air was performed to obtain the final values of the OTP. The SR measurement was performed at $0 \%$ air (100 $\% \mathrm{~N}_{2}$ ), $30 \%$ air, $50 \%$ air and $T_{1}^{-1}$ was plotted as function of \% air to estimate the OTP as shown in Fig. 8B. This approach was used earlier to estimate OTP in model and biological membranes $49,56,57$.

\section{Results}

\subsection{Effect of Incubation Time on the Binding of $\alpha$-Crystallin and the Membrane Surface Occupied by $\alpha$ - Crystallin}

To examine the time dependence of binding of $\alpha$-crystallin with membrane, $35 \mu \mathrm{M}$ of $\alpha$-crystallin was incubated with $9.4 \mathrm{mM}$ of POPC in total volume of $70 \mu \mathrm{L}$ of Hepes buffer. The incubation was done at $37^{\circ} \mathrm{C}$ for up to $16 \mathrm{hr}$ with gentle shaking. The samples were transferred to TPX capillary at different incubation times and the CW EPR measurements were performed. Fig. 2A shows the normalized EPR spectra of CSL in membrane with $\alpha$-crystallin binding at different incubation times. Low field EPR line signal intensity decreases with an increased incubation time and saturates at about $8 \mathrm{hrs}$ as demonstrated in Fig. 2B. EPR spectra at $0 \mathrm{hr}$ (Fig. 2) was used as control representing the EPR signal for membrane alone (membrane was incubated without $\alpha$-crystallin) and thus represents the unbound contribution. EPR spectra at different incubation times (concentration of $\alpha$-crystallin and POPC is fixed) represent the bound plus unbound contribution. Since our EPR spin label CSL is near the membrane surface and the $\alpha$-crystallin is bound on the membrane surface, the percentage of CSL spin label affected on the membrane surface by the binding of $\alpha$-crystallin is estimated as:

\% CSL spin label affected $=\left\{\left\{\mathrm{U}_{0}-\left(\mathrm{B}_{0}+\mathrm{U}_{\mathrm{o}}\right)\right\} /\left(\mathrm{U}_{0}\right)\right\}(100 \%)$

where $U_{0}$ is the low field EPR line peak to peak signal intensity from unbound contribution and $\left(B_{0}+U_{0}\right)$ is the low field EPR line peak to peak signal intensity from bound plus unbound contribution (see Fig. 2C). Because our membranes are SUVs of $\sim 30 \mathrm{~nm} \stackrel{44}{ }$, approximately $60 \%$ of the CSL spin labels are in the outer membrane surface and $40 \%$ of the CSL spin labels are in the inner membrane surface. Only the spin labels that are on the outer membrane surface are affected by binding of $\alpha$-crystallin. So, the corrected \% spin labels are affected or the \% of outer membrane surface occupied by $\alpha$-crystallin is obtained by multiplying equation (3) by the correction factor (100/60):

$\%$ membrane surface occupied $=\{\%$ CSL spin label affected $\}(100 / 60)$

Using equation (4), the percentage of the membrane surface occupied by $\alpha$-crystallin at different incubation times is obtained. Fig 3. shows that percentage of membrane surface occupied by $\alpha$-crystallin increases with an increase of incubation time. This indicates that binding increases with incubation time and reaches a maximum at about 8 hours (see Fig. 3).

\subsection{Ka of a-Crystallin to Phospholipid Membrane}

The $K_{a}$ of $\alpha$-crystallin with the POPC membrane was determined by varying the concentration of $\alpha$-crystallin and by keeping the concentration of POPC fixed. POPC concentration was fixed at $9.4 \mathrm{mM}$ and $\alpha$-crystallin concentration was varied from 0 to $53 \mu \mathrm{M}$. The $\alpha$-crystallin and the membrane mixture were incubated for 16 hrs at $37^{\circ} \mathrm{C}$ with gentle shaking. Fig. 4A shows normalized CW EPR spectra for the CSL in membrane with varying concentrations of $\alpha$ crystallin. Low field EPR line signal intensity decreases with an increase in $\alpha$-crystallin concentration and saturates at about $18 \mu \mathrm{M}$ as demonstrated in Fig. 4B. Peak to peak signal intensity of low field EPR line at $0 \mu \mathrm{M} \alpha$-crystallin concentration was used as control representing the unbound contribution $\left(\mathrm{U}_{\mathrm{o}}\right)$. Similarly peak to peak signal intensity of low field EPR line at $0.07,0.31,1.40,18,35$, and $53 \mu \mathrm{M} \alpha$-crystallin concentrations represent the contribution from bound plus unbound $\left(B_{0}+U_{o}\right)$. Fig. 5 shows the percentage membrane surface occupied by $\alpha$-crystallin at different $\alpha$ crystallin concentrations and is obtained by using equation 4 and Fig. 4. Since the outer membrane surface is identical throughout the vesicle, $\alpha$-crystallin can bind to any region of the membrane surface with equal probability indicating 
This is an author-produced, peer-reviewed version of this article. The final, definitive version of this document can be found online at Current Eye Research, published by Taylor \& Francis. Copyright restrictions may apply. doi: 10.1080/02713683.2020.1786131

that there is only one possible binding site, so we have used one-site ligand binding function to estimate $\mathrm{K}_{\mathrm{a}}$. Data points in Fig. 5 are fitted with one-site ligand binding function and $K_{a}$ is estimated to be $4.9 \pm 2.4 \mu M^{-1}$. One-site ligand binding function was used earlier to estimate $K_{a}$ for saturation binding curves $\frac{58,59}{}$. Previously, Mulders et al. $\underline{37}$ labelled $\alpha$-crystallin with $\left[{ }^{35} \mathrm{~S}\right]$ methionine and estimated $\mathrm{K}_{\mathrm{a}}$ of $7.69 \mu \mathrm{M}^{-1}$ for $\alpha$-crystallin with alkali-washed lens plasma membrane.

Also from Fig. 5, the maximum membrane surface occupied by $\alpha$-crystallin for POPC membrane is about $10 \%$ and the binding is saturable. To the best of our knowledge, our attempt is the first to estimate the membrane surface occupied by $\alpha$-crystallin. Previously, Mulders et al. $\frac{37}{3}$ labelled $\alpha$-crystallin with $\left[{ }^{35} \mathrm{~S}\right.$ ] methionine and incubated with various concentrations of the egg yolk lecithin vesicle (i.e., phosphatidylcholine vesicle) and found about $\sim 10 \% \alpha$ crystallin bound. Several groups have investigated the binding of $\alpha$-crystallin with phospholipid vesicles (protein free vesicle) $24,34-36,38$. Tang et al. $\frac{38}{}$ and Borchman et al. ${ }^{34}$ used fluorophore NBD-PE to investigate the binding of $\alpha-$ crystallin with protein free vesicle, and they observed the saturable binding. Similarly, Cobb et al..$^{\frac{35}{5}}$ conjucated $\alpha-$ crystallin to the AlexaFluor $350^{\mathrm{TM}}$ fluorescent tag and found saturable binding for protein free vesicle.

\subsection{Mobility of Membrane After Binding of $\alpha$-Crystallin}

Fig. 6A shows the representative CW EPR spectra of CSL in POPC membrane incubated with $53 \mu \mathrm{M}$ of $\alpha$-crystallin for $16 \mathrm{hrs}$ at $37^{\circ} \mathrm{C}$. The concentration of POPC was fixed at $9.4 \mathrm{mM}$. The values used for the calculation of mobility parameter $\stackrel{60}{ }, h_{+}$and $h_{0}$, are measured directly from the spectra as shown in figure 6 . The mobility parameter $\left(h_{+} / h_{0}\right)$ includes contribution of both orientation and rotational mobility of the CSL molecules $\underline{60}$. We have used the mobility parameter previously to estimate the mobility of the membrane at varying cholesterol concentrations $\frac{56,61,62}{6}$. Fig. $6 \mathrm{~B}$ shows the profiles of mobility parameter with varying concentrations of $\alpha$-crystallin. Mobility parameter decreases sharply with an increase in $\alpha$-crystallin concentration and saturates at about $18 \mu \mathrm{M}$. This indicates the rate of motion of the membrane decreases as more and more $\alpha$-crystallin binds the membrane. Because the nitroxide moiety of CSL is located near the membrane surface (see Fig. 1), it reflects orientation and rotational motion near the membrane surface. This indicates the binding of $\alpha$-crystallin makes the membrane more immobilized near the head group region of the PL.

Previously, we have used CSL spin label in POPC membrane with varying cholesterol concentrations ( 0 mol \% to 75 $\mathrm{mol} \%)$ to investigate the mobility of POPC membrane in the presence of cholesterol $\frac{63}{}$. We also saw a sharp decrease

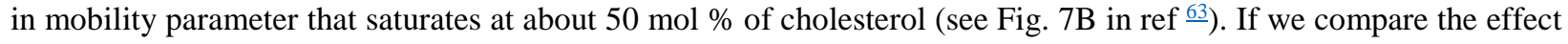
of $\alpha$-crystallin binding in membrane mobility vs the effect of cholesterol in membrane mobility, then the decrease in membrane mobility by increasing $\alpha$-crystallin concentration from $0 \mu \mathrm{M}$ to $18 \mu \mathrm{M}$ is comparable to the decrease in membrane mobility by increasing cholesterol concentration from $33 \mathrm{~mol} \%$ to $66 \mathrm{~mol} \%$ (see Fig. 7B in ref $\underline{63}$ for cholesterol data).

Borchman et al. ${ }^{34}$ used fluorophore NBD-PE that partitions near the membrane head group region to investigate the binding of $\alpha$-crystallin to bovine lens lipid vesicles. They found that the head group region becomes less mobile with binding of $\alpha$-crystallin $\frac{34}{4}$, which is in agreement with our findings.

\subsection{Fluidity and OTP of Membrane After Binding of $\alpha$-Crystallin}

The spin-lattice relaxation rate $\left(T_{1}^{-1}\right)$ obtained from the SR EPR measurements of lipid spin labels in deoxygenated samples depends primarily on the rotational correlation time of the nitroxide moiety within the lipid bilayer $\underline{64}, \underline{65}$. Thus, $T_{1}^{-1}$ can be used as a convenient quantitative measure of membrane fluidity that reflects the local motional properties of the lipid alkyl chain $\underline{62,66-69}$. Previously, we have used $T_{1}^{-1}$ as a convenient quantitative measure of fluidity using phospholipid analog (n-PC, 9-SASL) and cholesterol analog (CSL) spin labels in model and eye lens lipid membranes $56,62,66-70$. Representative saturation recovery EPR signal of CSL in membrane incubated with $0 \mu \mathrm{M} \alpha$-crystallin and $53 \mu \mathrm{M} \alpha$-crystallin are shown in Fig. 7 (The concentration of POPC was fixed to $9.4 \mathrm{mM}$ ). Measurements were performed for deoxygenated samples $\left(100 \% \mathrm{~N}_{2}\right)$ and samples equilibrated with selected air/nitrogen mixture. All saturation-recovery EPR signals shown in Fig. 7 were satisfactorily fit to a single exponential function. As can be seen from residuals (the experimental signal minus the fitting curve) in all cases, the single-exponential fit was excellent. 
This is an author-produced, peer-reviewed version of this article. The final, definitive version of this document can be found online at Current Eye Research, published by Taylor \& Francis. Copyright restrictions may apply. doi: 10.1080/02713683.2020.1786131

$T_{1}^{-1}$ 's obtained from the deoxygenated samples for varying $\alpha$-crystallin concentrations allows us to construct fluidity profiles as shown in Fig. 8A. Fig. 8A shows that membrane fluidity decreases with an increase in $\alpha$-crystallin concentration. This indicates that as more and more $\alpha$-crystallin binds the membrane, the membrane becomes less mobile near the head group region of the PL. This finding is in agreement with mobility measurement presented in Section 3.3.

Previously, we have observed with CSL spin label in the membrane that membrane fluidity decrease with increase in cholesterol concentration from $0 \mathrm{~mol} \%$ to $50 \mathrm{~mol} \%$ (see Fig. 7C in ref $\frac{63}{3}$ ). If we compare the decrease in membrane fluidity by $\alpha$-crystallin binding in membrane vs the decrease in membrane fluidity by increasing cholesterol content from $0 \mathrm{~mol} \%$ to $50 \mathrm{~mol} \%$ in membrane. Then the overall change in $T_{1}^{-1}$ value is $0.031 \mu \mathrm{s}^{-1}$ (see. Fig. 8A) with $\alpha-$

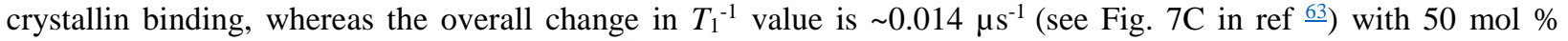
cholesterol content increase. The change in $T_{1}^{-1}$ is about twice as big (double) with $\alpha$-crystallin binding in comparison with increasing cholesterol content to $50 \mathrm{~mol} \%$, which indicates that the effect on membrane fluidity is greater with $\alpha$-crystallin binding in comparison with $50 \mathrm{~mol} \%$ cholesterol content increase in membrane.

SR EPR measurements were taken at $0 \%$ air (i.e., $100 \% \mathrm{~N}_{2}$ ), $30 \%$ air, and $50 \%$ air and then extrapolation to $100 \%$ air is performed to obtain the final values of the OTPs. The theory for the OTP is described in Sect. 2.6. Representative SR signals at $100 \% \mathrm{~N}_{2}$ and $50 \%$ air is shown in Fig. 7. Fig. 8C shows the profiles of OTP in the membrane with varying concentrations of $\alpha$-crystallin. The OTP decreases with an increase with $\alpha$-crystallin concentration which indicates that the binding of $\alpha$-crystallin forms a barrier to oxygen transport. This barrier increases as more and more $\alpha$-crystallin binds with the membrane. Our results are in strong agreement with the barrier hypothesis proposed earlier 5,14, $\underline{2}$, where binding of $\alpha$-crystallin to membrane occludes membrane pores forming a barrier to diffusion. As proposed earlier, blocking of membrane pores could induce oxidative conditions in the lens center which leads to the development of nuclear cataract $5,14,32$.

\section{Discussion}

Several studies have reported that with age and cataract formation the amount of membrane bound $\alpha$-crystallin increases $14,15, \underline{28,29}, \underline{32}$. A recent clinical study has also shown that if the level of unbound $\alpha$-crystallin in the lens is higher, then there is a lower risk to have lens opacification or need for the cataract surgery $\underline{29}$. Clinical study further confirmed that $\alpha$-crystallin levels decrease over time with age and this decrease in $\alpha$-crystallin leads to cataract $\frac{29}{}$. The decreased $\alpha$-crystallin level in lens cytoplasm will increase the amount of membrane bound $\alpha$-crystallin which increases light scattering and induces cataract formation. So, it is very crucial to understand the mechanism on the fundamental level that promotes or inhibits $\alpha$-crystallin to bind the lens membranes.

Previously, Tang et. $\mathrm{al}^{38}$ investigated the binding of $\alpha$-crystallin to disterolphosphatidylcholine (DSPC), sphingomyelin (SM), and egg-PC membrane. They found that the binding $\alpha$-crystallin depends upon the hydrocarbon chain order. Cobb et. al $\frac{35}{2}$ investigated the binding of $\alpha$-crystallin to lipids with varying lipid structural order and saturation of acyl chain. Their finding suggests that the binding of $\alpha$-crystallin to lipid membrane is independent of lipid bilayer fluidity and over-all structural order. Borchman et. al $\stackrel{34}{4}$ investigated the binding of $\alpha$-crystallin with bovine lens lipids and found that lipid headgroups become less mobile with binding which is in agreement with our data presented here. The florescence approaches previously employed utilized the fluorophore NBD-PE which resides on the polar head group region of the membrane $\underline{38}$ or the $\alpha$-crystallin was conjugated to the Alexfluor $350^{\mathrm{TM}}$ fluorescent tag $\frac{35}{5}$, to investigate the binding of $\alpha$-crystallin to lipid membrane. As mentioned in the previous study, these florescence approaches include ultracentrifugation steps where bound $\alpha$-crystallin pellet was used for analysis. These techniques may result in incomplete pelleting of the vesicles that could underestimate the amount of bound $\alpha$ crystallin $\underline{35}$. Here we have developed the EPR approach where the CSL spin label is located near the surface of the membrane and gives the information about the binding of $\alpha$-crystallin as well as the physical properties of membrane after the binding of $\alpha$-crystallin. The EPR approach avoids the centrifugation steps and has the unique ability to give the information about the membrane surface occupied by $\alpha$-crystallin.

In addition, the EPR approach also gives new information about the mobility, fluidity, and OTP of the membrane after $\alpha$-crystallin binding. As discussed in Sect. 3.4, OTP gives the information about barrier in the membrane and this barrier increases with $\alpha$-crystallin binding. Although the study performed here was for the protein free membrane, we can speculate that binding of $\alpha$-crystallin to lens membrane will occlude the pores of aquaporin-0 (AQP0) and connexins (Cx46 and Cx50), the most abundant integral transmembrane proteins in human lens fiber cell membranes $\underline{71-73}$. AQP0 control transport of water and some neutral solutes ${ }^{74}$. Connexins form gap junctions between lens fiber 
This is an author-produced, peer-reviewed version of this article. The final, definitive version of this document can be found online at Current Eye Research, published by Taylor \& Francis. Copyright restrictions may apply. doi: 10.1080/02713683.2020.1786131

cells and control the exchange of ions and small metabolites between lens cells $\frac{75}{7}$. Thus, occluding the membrane pores would result in a barrier to diffusion of these small molecules which ultimately could lead to the development of nuclear cataracts ${ }^{14}$.

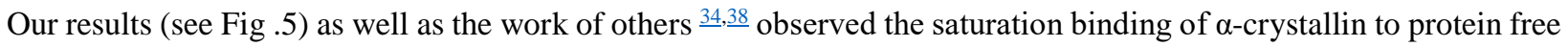
lipid vesicles. For the POPC membranes, the phospholipid head group has a neutral charge and the interior hydrocarbon chains are hydrophobic. As reported earlier ${ }^{37,76} \alpha$-crystallin has a hydrophobic regions on its surface, thus it is likely that the binding of $\alpha$-crystallin to the membrane is lipid-specific and is through hydrophobic interaction. Previously, Tang et. al $\underline{38}$ reported that the binding of $\alpha$-crystallin to protein free membranes depends on the hydrocarbon chain order, Cobb and Petrash ${ }_{27}^{27}$ postulated that the binding of $\alpha$-crystallin to lens membrane is predominantly through hydrophobic interactions, and Ifeanyi and Takemoto ${ }^{77}$ demonstrated that the binding of $\alpha$ crystallin to lens membrane is specific and saturable.

$\alpha$-crystallin consists of $\alpha \mathrm{A}$ - and $\alpha \mathrm{B}$ - crystallin subunits with $3 \alpha \mathrm{A}$ to $1 \alpha \mathrm{B}$ molar ratio in adult bovine lens $\frac{18}{.} \alpha$ crystallin forms highly polydisperse oligomers $\underline{18,41}$ in which subunits are known to exchange between the oligomer 41,78-80. This oligomer are a dynamic system where the size of the oligomer changes dynamically by adding or removing the monomer or small oligomers $\stackrel{41,81}{ }$. Srinivas et. al $\stackrel{82}{ }$ studied the thermal stability of $\alpha$-crystallin using differential scanning calorimetry and found that the unfolding temperature between $58-61^{\circ} \mathrm{C}$ for $3: 1$ heteropolymer and aA-crystallin homopolymer and also observe no precipitation upon thermal unfolding. Based on this thermodynamic stability of $\alpha$-crystallin, it is very likely that the soluble $\alpha$-crystallin (not bound to the membrane) is stable at $37{ }^{\circ} \mathrm{C}$ for 16 hours. We think that the $\alpha$-crystallin that is in the solution after binding saturation is achieved will interact with membrane bound $\alpha$-crystallin via subunit exchange. However looking at the higher binding affinity values (Fig .5) we speculate that the subunit exchange takes place only between the soluble $\alpha$-crystallin and solvent exposed subunits of membrane bound alpha-crystallin. Previously, Cobb and Petrash $\underline{16}$ used fluorescence resonance energy transfer and demonstrated that the subunit exchange takes between solvent exposed regions of membrane bound $\alpha$-crystallin and the soluble $\alpha$-crystallin.

$\alpha$-crystallin belongs to the small heat shock protein family and serves as chaperone, binding with the denatured proteins preventing their aggregation $\underline{78,79,82}$. Subunit exchange between the $\alpha$-crystallin oligomers is required to main the chaperone like activity $\underline{16, \underline{78}}$. It has been reported that the subunit exchange depends upon temperature $\underline{79}, \underline{80}$ and reached complete equilibrium within 4 hour for alpha $\alpha$ A-crystallin subunits $\underline{79}$ whereas for mixing of isolated bovine $\alpha \mathrm{A}$ and $\alpha \mathrm{B}$ crystallin the exchange reaction continues until 24 hours $^{80}$. For $\alpha \mathrm{A}$-crystallin subunit exchange, the rate

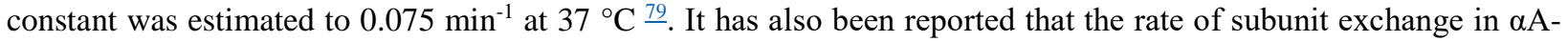
crystallin changes depending upon the size and concentration of polypeptide bound to the $\alpha \mathrm{A}$-crystallin $\underline{79}$. Based on these earlier findings, we speculate that when binding of $\alpha$-crystallin to membranes increases with time (Fig. 3), the rate of subunit exchange between membrane bound $\alpha$-crystallin and soluble $\alpha$-crystallin may change.

The CSL spin labels are located in both regions in the membrane i.e. in the region where $\alpha$-crystallin is bound to the membrane and in the region where $\alpha$-crystallin is not bound in the membrane. Therefore, we expect to have two values of the $T_{1}{ }^{-1}$ 's from these two regions; however, we get only one average $T_{1}{ }^{-1}$ value (see Fig. 8A). This indicates that the exchange rate of CSL spin labels in membranes between the bound $\alpha$-crystallin and unbound $\alpha$-crystallin is greater than $T_{1}{ }^{-1}$ giving an averaged $T_{1}^{-1}$. This exchange rate between these bound and unbound $\alpha$-crystallin regions in the membrane is faster when compared with the spin-lattice relaxation rate of lipid spin labels in membranes between $10^{5}$ and $10^{6} \mathrm{~s}^{-1}$ ( $T_{1}$ from 1 to $10 \mu \mathrm{s}$ ). This faster exchange rate implies that the bound $\alpha$-crystallin membrane regions should be small, allowing us to hypothesize that it is likely that the $\alpha$-crystallin monomer or smaller oligomers with few subunits are participating in binding rather than the larger oligomers of $\alpha$-crystallin.

Autoxidation of the phospholipid membrane is very slow process and it depends on the number of double bonds on the acyl chain $\underline{83}$. The accumulation of phospholipid peroxidation products was reported earlier using a thiobarbituric acid assay for autoxidation of 1-palmitoyl-2-arachidonoyl-phosphatidylcholine (PAPC) membrane $\underline{83}$. The phospholipid peroxidation products was very low for the first 75 hours of autoxidation of PAPC membrane $\frac{83}{\text {. In the }}$ PAPC molecule, there are four double bonds in one acyl chain (arachidonic) and the second acyl chain (palmitic) is fully saturated whereas in the case of the POPC molecule that is used in this project, has only one double bond in one acyl chain (oleic) and the second chain (palmitic) is fully saturated. Thus, it is very likely that POPC autoxidation will not occur in 16-hour incubation at $37^{\circ} \mathrm{C}$. We measured the EPR signal of CSL in POPC membrane ( 30 nm SUVs) at 0 hour and after 16-hour incubation at $37^{\circ} \mathrm{C}$ and found that there is no significant change in the EPR signal indicating the stability of the SUVs. 
This is an author-produced, peer-reviewed version of this article. The final, definitive version of this document can be found online at Current Eye Research, published by Taylor \& Francis. Copyright restrictions may apply. doi: 10.1080/02713683.2020.1786131

\section{Conclusions}

In this study, we have demonstrated the EPR approach to estimate $K_{a}$ of $\alpha$-crystallin to phospholipid membranes. The unique ability of the EPR approach to get the information about the membrane surface occupied by $\alpha$-crystallin is also demonstrated. In addition, the physical properties of membrane after the binding of $\alpha$-crystallin were also obtained. The physical properties include mobility parameter, fluidity parameter and OTP which decrease as more and more $\alpha$ crystallin binds to the membrane. All these findings contribute to better undersetting of $\alpha$-crystallin association with the membrane at the molecular level.

\section{Declaration of Interest}

The authors report no conflicts of interest.

Research reported in this publication was supported by the National Institutes of Health (USA) under Grants R01 EY030067, R01 EY015526, and P41 EB001980.

\section{References}

1. Hejtmancik JF, Kantorow M. Molecular genetics of age-related cataract. Exp Eye Res 2004;79:3-9.

2. Shiels A, Hejtmancik JF. Genetic origins of cataract. Arch Ophthalmol 2007;125:165-173.

3. Uwineza A, Kalligeraki AA, Hamada N, Jarrin M, Quinlan RA. Cataractogenic load - A concept to study the contribution of ionizing radiation to accelerated aging in the eye lens. Mutat Res 2019;779:68-81.

4. Bron AJ, Vrensen GF, Koretz J, Maraini G, Harding JJ. The ageing lens. Ophthalmologica 2000;214:86104.

5. $\quad$ Truscott RJ. Age-related nuclear cataract-oxidation is the key. Exp Eye Res 2005;80:709-725.

6. Hsuan JD, Brown NA, Bron AJ, Patel CK, Rosen PH. Posterior subcapsular and nuclear cataract after vitrectomy. J Cataract Refract Surg 2001;27:437-444.

7. Chung CP, Hsu SY, Wu WC. Cataract formation after pars plana vitrectomy. Kaoh J Med Sci 2001;17:8489.

8. Huang L, Estrada R, Yappert MC, Borchman D. Oxidation-induced changes in human lens epithelial cells. 1. Phospholipids. Free Radic Biol Med 2006;41:1425-1432.

9. $\quad$ Palmquist BM, Philipson B, Barr PO. Nuclear cataract and myopia during hyperbaric oxygen therapy. Br J Ophthalmol 1984;68:113-117.

10. Freel CD, Gilliland KO, Mekeel HE, Giblin FJ, Costello MJ. Ultrastructural characterization and Fourier analysis of fiber cell cytoplasm in the hyperbaric oxygen treated guinea pig lens opacification model. Exp Eye Res 2003;76:405-415.

11. Truscott RJ, Augusteyn RC. Changes in human lens proteins during nuclear cataract formation. Exp Eye Res 1977;24:159-170.

12. Moreau KL, King JA. Protein misfolding and aggregation in cataract disease and prospects for prevention. Trends Mol Med 2012;18:273-282.

13. Zhao L, Chen XJ, Zhu J, Xi YB, Yang X, Hu LD, Ouyang H, Patel SH, Jin X, Lin D, et al. Corrigendum: Lanosterol reverses protein aggregation in cataracts. Nature 2015;526:595.

14. Friedrich MG, Truscott RJ. Large-scale binding of alpha-crystallin to cell membranes of aged normal human lenses: a phenomenon that can be induced by mild thermal stress. Invest Ophthalmol Vis Sci 2010;51:5145-5152.

15. Chandrasekher G, Cenedella RJ. Protein associated with human lens 'native' membrane during aging and cataract formation. Exp Eye Res 1995;60:707-717.

16. Cobb BA, Petrash JM. alpha-Crystallin chaperone-like activity and membrane binding in age-related cataracts. Biochemistry 2002;41:483-490.

17. Chandrasekher G, Cenedella RJ. Protein associated with human lens 'native' membrane during aging and cataract formation. Exp Eye Res 1995;60:707-717.

18. Horwitz J, Bova MP, Ding LL, Haley DA, Stewart PL. Lens alpha-crystallin: function and structure. Eye (Lond) 1999;13 ( Pt 3b):403-408.

19. Santhoshkumar P, Raju M, Sharma KK. alphaA-crystallin peptide SDRDKFVIFLDVKHF accumulating in aging lens impairs the function of alpha-crystallin and induces lens protein aggregation. PLoS One 2011;6:e19291.

20. Horwitz J. Alpha-crystallin. Exp Eye Res 2003;76:145-153. 
This is an author-produced, peer-reviewed version of this article. The final, definitive version of this document can be found online at Current Eye Research, published by Taylor \& Francis. Copyright restrictions may apply. doi: 10.1080/02713683.2020.1786131

21. Nagaraj RH, Nahomi RB, Mueller NH, Raghavan CT, Ammar DA, Petrash JM. Therapeutic potential of alpha-crystallin. Biochim Biophys Acta 2016;1860:252-257.

22. Boyle DL, Takemoto L. EM immunolocalization of alpha-crystallins: association with the plasma membrane from normal and cataractous human lenses. Curr Eye Res 1996;15:577-582.

23. Cenedella RJ, Fleschner CR. Selective association of crystallins with lens 'native' membrane during dynamic cataractogenesis. Curr Eye Res 1992;11:801-815.

24. Grami V, Marrero Y, Huang L, Tang D, Yappert MC, Borchman D. alpha-Crystallin binding in vitro to lipids from clear human lenses. Exp Eye Res 2005;81:138-146.

25. Tang D, Borchman D, Yappert MC, Cenedella RJ. Influence of cholesterol on the interaction of alphacrystallin with phospholipids. Exp Eye Res 1998;66:559-567.

26. Chandrasekher G, Cenedella RJ. Properties of alpha-crystallin bound to lens membrane: probing organization at the membrane surface. Exp Eye Res 1997;64:423-430.

27. Cobb BA, Petrash JM. Characterization of alpha-crystallin-plasma membrane binding. J Biol Chem 2000;275:6664-6672.

28. Su SP, McArthur JD, Friedrich MG, Truscott RJ, Aquilina JA. Understanding the alpha-crystallin cell membrane conjunction. Mol Vis 2011;17:2798-2807.

29. Datiles MB, 3rd, Ansari RR, Yoshida J, Brown H, Zambrano AI, Tian J, Vitale S, Zigler JS, Jr., Ferris FL, 3rd, West SK, et al. Longitudinal Study of Age-Related Cataract Using Dynamic Light Scattering: Loss of alpha-Crystallin Leads to Nuclear Cataract Development. Ophthalmology 2016;123:248-254.

30. Cenedella RJ, Fleschner CR. Selective association of crystallins with lens 'native' membrane during dynamic cataractogenesis. Curr Eye Res 1992;11:801-815.

31. Tang D, Borchman D, Yappert MC. Alpha-crystallin/lens lipid interactions using resonance energy transfer. Ophthalmic Res 1999;31:452-462.

32. Friedrich MG, Truscott RJ. Membrane association of proteins in the aging human lens: profound changes take place in the fifth decade of life. Invest Ophthalmol Vis Sci 2009;50:4786-4793.

33. Sato H, Borchman D, Ozaki Y, Lamba OP, Byrdwell WC, Yappert MC, Paterson CA. Lipid-protein interactions in human and bovine lens membranes by Fourier transform Raman and infrared spectroscopies. Exp Eye Res 1996;62:47-53.

34. Borchman D, Tang D. Binding capacity of alpha-crystallin to bovine lens lipids. Exp Eye Res 1996;63:407-410.

35. Cobb BA, Petrash JM. Factors influencing alpha-crystallin association with phospholipid vesicles. Mol Vis 2002;8:85-93.

36. Ifeanyi F, Takemoto L. Interaction of lens crystallins with lipid vesicles. Exp Eye Res 1991;52:535-538.

37. Mulders JW, Stokkermans J, Leunissen JA, Benedetti EL, Bloemendal H, de Jong WW. Interaction of alpha-crystallin with lens plasma membranes. Affinity for MP26. Eur J Biochem 1985;152:721-728.

38. Tang D, Borchman D, Yappert MC, Cenedella RJ. Influence of cholesterol on the interaction of alphacrystallin with phospholipids. Exp Eye Res 1998;66:559-567.

39. Ward KA. Alpha-crystallin incorporates to form proteoliposomes. Invest Ophthalmol Vis Sci 1996;37:S599.

40. Deeley JM, Mitchell TW, Wei X, Korth J, Nealon JR, Blanksby SJ, Truscott RJ. Human lens lipids differ markedly from those of commonly used experimental animals. Biochim Biophys Acta 2008;1781:288-298.

41. Ryazantsev SN, Poliansky NB, Chebotareva NA, Muranov KO. 3D structure of the native alpha-crystallin from bovine eye lens. Int JBiol Macromol 2018;117:1289-1298.

42. Horwitz J, Huang QL, Ding L, Bova MP. Lens alpha-crystallin: chaperone-like properties. Methods Enzymol 1998;290:365-383.

43. Lampi KJ, Fox CB, David LL. Changes in solvent accessibility of wild-type and deamidated betaB2crystallin following complex formation with alphaA-crystallin. Exp Eye Res 2012;104:48-58.

44. Mainali L, Vasquez-Vivar J, Hyde JS, Subczynski WK. Spin-labeled small unilamellar vesicles with the T1-sensitive saturation-recovery EPR display as an oxygen sensitive analyte for measurement of cellular respiration. Appl Magn Reson 2015;46:885-895.

45. Buboltz JT. A more efficient device for preparing model-membrane liposomes by the rapid solvent exchange method. Rev Sci Instrum 2009;80:124301.

46. Buboltz JT, Feigenson GW. A novel strategy for the preparation of liposomes: rapid solvent exchange. Biochim Biophys Acta 1999;1417:232-245.

47. Subczynski WK, Felix CC, Klug CS, Hyde JS. Concentration by centrifugation for gas exchange EPR oximetry measurements with loop-gap resonators. J Magn Reson 2005;176:244-248. 
This is an author-produced, peer-reviewed version of this article. The final, definitive version of this document can be found online at Current Eye Research, published by Taylor \& Francis. Copyright restrictions may apply. doi: 10.1080/02713683.2020.1786131

48. Mainali L, Camenisch TG, Hyde JS, Subczynski WK. Saturation recovery EPR spin-labeling method for quantification of lipids in biological membrane domains. Appl Magn Reson 2017;48:1355-1373.

49. Subczynski WK, Hyde JS, Kusumi A. Oxygen permeability of phosphatidylcholine-cholesterol membranes. Proc Natl Acad Sci USA 1989;86:4474-4478.

50. Yin JJ, Subczynski WK. Effects of lutein and cholesterol on alkyl chain bending in lipid bilayers: a pulse electron spin resonance spin labeling study. Biophys J 1996;71:832-839.

51. Kusumi A, Subczynski WK, Hyde JS. Oxygen transport parameter in membranes as deduced by saturation recovery measurements of spin-lattice relaxation times of spin labels. Proc Natl Acad Sci USA 1982;79:1854-1858.

52. Windrem DA, Plachy WZ. The diffusion-solubility of oxygen in lipid bilayers. Biochim Biophys Acta 1980;600:655-665.

53. Hyde JS, Subczynski WK. Simulation of EPR spectra of the oxygen-sensitive spin-label probe CTPO. J Magn Res 1984;56.

54. Hyde JS, Subczynski WK. Spin-label oximetry. In: Berliner LJ, Reuben J, editors. Biological Magnetic Resonance. New York: Plenum Press; 1989. pp 399-425.

55. Subczynski WK, Hyde JS. Diffusion of oxygen in water and hydrocarbons using an electron spin resonance spin-label technique. Biophys J 1984;45:743-748.

56. Raguz M, Mainali L, Widomska J, Subczynski WK. Using spin-label electron paramagnetic resonance (EPR) to discriminate and characterize the cholesterol bilayer domain. Chem Phys Lipids 2011;164:819829.

57. Mainali L, O'Brien WJ, Subczynski WK. Detection of cholesterol bilayer domains in intact biological membranes: Methodology development and its application to studies of eye lens fiber cell plasma membranes. Exp Eye Res 2019;178:72-81.

58. Schultz KM, Feix JB, Klug CS. Disruption of LptA oligomerization and affinity of the LptA-LptC interaction. Protein Sci 2013;22:1639-1645.

59. Schultz KM, Lundquist TJ, Klug CS. Lipopolysaccharide binding to the periplasmic protein LptA. Protein Sci 2017;26:1517-1523.

60. Schreier S, Polnaszek CF, Smith IC. Spin labels in membranes. Problems in practice. Biochim Biophys Acta 1978;515:395-436.

61. Raguz M, Mainali L, Widomska J, Subczynski WK. The immiscible cholesterol bilayer domain exists as an integral part of phospholipid bilayer membranes. Biochim Biophys Acta 2011;1808:1072-1080.

62. Mainali L, Raguz M, Subczynski WK. Phases and domains in sphingomyelin-cholesterol membranes: structure and properties using EPR spin-labeling methods. Eur Biophys J 2012;41:147-159.

63. Raguz M, Mainali L, Widomska J, Subczynski WK. Using spin-label electron paramagnetic resonance (EPR) to discriminate and characterize the cholesterol bilayer domain. Chem Phys Lipids 2011;164:819829.

64. Robinson BH, Haas DA, Mailer C. Molecular dynamics in liquids: spin-lattice relaxation of nitroxide spin labels. Science 1994;263:490-493.

65. Mailer C, Nielsen RD, Robinson BH. Explanation of spin-lattice relaxation rates of spin labels obtained with multifrequency saturation recovery EPR. J Phys Chem A 2005;109:4049-4061.

66. Mainali L, Raguz M, Camenisch TG, Hyde JS, Subczynski WK. Spin-label saturation-recovery EPR at Wband: applications to eye lens lipid membranes. J Magn Reson 2011;212:86-94.

67. Mainali L, Feix JB, Hyde JS, Subczynski WK. Membrane fluidity profiles as deduced by saturationrecovery EPR measurements of spin-lattice relaxation times of spin labels. J Magn Reson 2011;212:418425.

68. Mainali L, Hyde JS, Subczynski WK. Using spin-label W-band EPR to study membrane fluidity profiles in samples of small volume. J Magn Reson 2013;226:35-44.

69. Subczynski WK, Raguz M, Widomska J. Studying lipid organization in biological membranes using liposomes and EPR spin labeling. Methods Mol Biol 2010;606:247-269.

70. Mainali L, Raguz M, O'Brien WJ, Subczynski WK. Properties of membranes derived from the total lipids extracted from the human lens cortex and nucleus. Biochim Biophys Acta 2013;1828:1432-1440.

71. Bassnett S, Shi Y, Vrensen GF. Biological glass: structural determinants of eye lens transparency. Philos Trans Royal Soc Lond Ser B Biol Sci 2011;366:1250-1264.

72. Gonen T, Cheng Y, Sliz P, Hiroaki Y, Fujiyoshi Y, Harrison SC, Walz T. Lipid-protein interactions in double-layered two-dimensional AQP0 crystals. Nature 2005;438:633-638. 
This is an author-produced, peer-reviewed version of this article. The final, definitive version of this document can be found online at Current Eye Research, published by Taylor \& Francis. Copyright restrictions may apply. doi: 10.1080/02713683.2020.1786131

73. Reichow SL, Gonen T. Lipid-protein interactions probed by electron crystallography. Curr Opin Struct Biol 2009;19:560-565.

74. $\quad$ Agre P. Nobel Lecture. Aquaporin water channels. Biosci Rep 2004;24:127-163.

75. Mathias RT, White TW, Gong X. Lens gap junctions in growth, differentiation, and homeostasis. Physiol Rev 2010;90:179-206.

76. Reddy GB, Kumar PA, Kumar MS. Chaperone-like activity and hydrophobicity of alpha-crystallin. IUBMB Life 2006;58:632-641.

77. Ifeanyi F, Takemoto L. Specificity of alpha crystallin binding to the lens membrane. Curr Eye Res 1990;9:259-265.

78. Bova MP, McHaourab HS, Han Y, Fung BK. Subunit exchange of small heat shock proteins. Analysis of oligomer formation of alphaA-crystallin and Hsp27 by fluorescence resonance energy transfer and sitedirected truncations. J Biol Chem 2000;275:1035-1042.

79. Bova MP, Ding LL, Horwitz J, Fung BK. Subunit exchange of alphaA-crystallin. J Biol Chem 1997;272:29511-29517.

80. van den Oetelaar PJ, van Someren PF, Thomson JA, Siezen RJ, Hoenders HJ. A dynamic quaternary structure of bovine alpha-crystallin as indicated from intermolecular exchange of subunits. Biochemistry 1990;29:3488-3493.

81. Horwitz J. Alpha-crystallin. Exp Eye Res 2003;76:145-153.

82. Srinivas P, Narahari A, Petrash JM, Swamy MJ, Reddy GB. Importance of eye lens alpha-crystallin heteropolymer with 3:1 alphaA to alphaB ratio: stability, aggregation, and modifications. IUBMB Life 2010;62:693-702.

83. Zareba M, Widomska J, Burke JM, Subczynski WK. Nitroxide free radicals protect macular carotenoids against chemical destruction (bleaching) during lipid peroxidation. Free Radic Biol Med 2016;101:446454.

Figure Captions

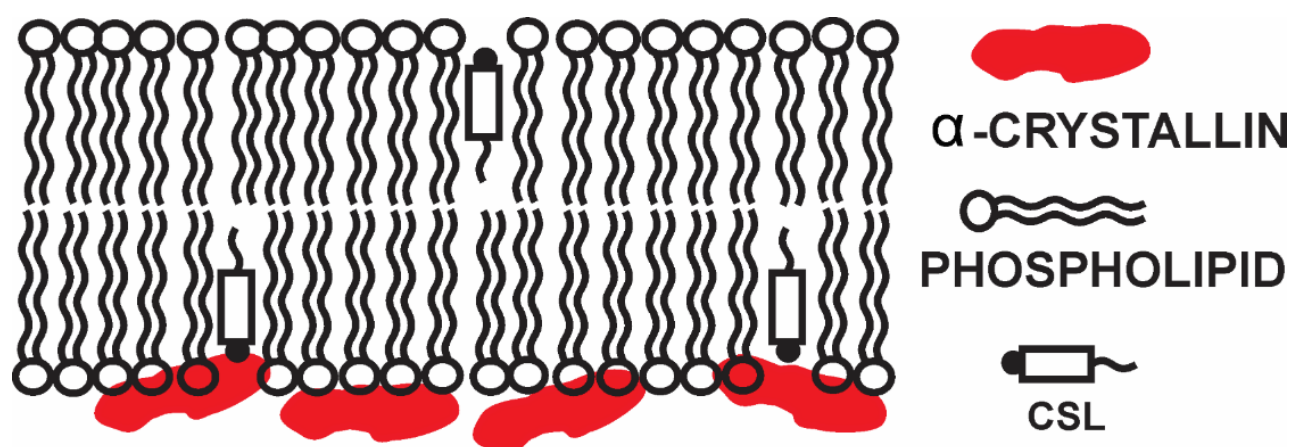

Figure 1. Schematic drawing showing the binding of $\alpha$ - crystallin in the membrane along with the approximate locations of cholesterol analogue spin label (CSL) in the membrane. The nitroxide moieties of spin labels are indicated by black dots. 
This is an author-produced, peer-reviewed version of this article. The final, definitive version of this document can be found online at Current Eye Research, published by Taylor \& Francis. Copyright restrictions may apply. doi: 10.1080/02713683.2020.1786131
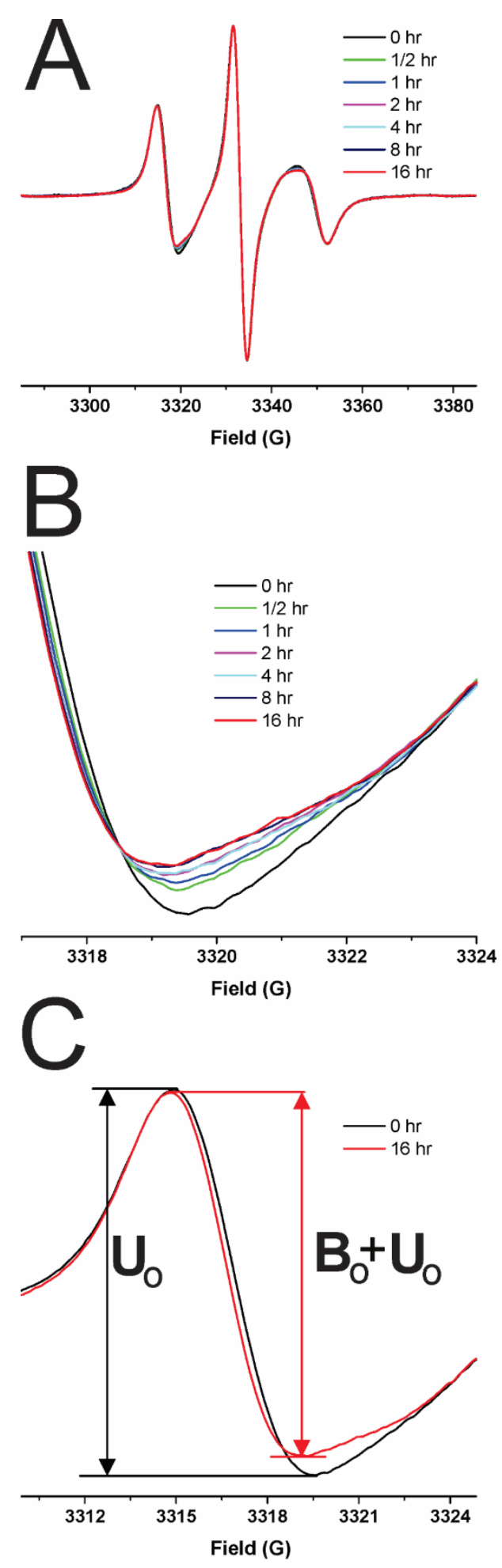

Figure 2. A) EPR spectra of CSL in membrane with $\alpha$-crystallin binding at different incubation time. All the samples were incubated at $37^{\circ} \mathrm{C}$. The concentration of POPC and $\alpha$-crystallin used was fixed at $9.4 \mathrm{mM}$ and $35 \mu \mathrm{M}$, respectively, in the total volume of $70 \mu \mathrm{L}$ (The sample at $0 \mathrm{hr}$ is membrane incubated without $\alpha$-crystallin and is used as a control). B) Fragment of EPR spectra demonstrating the change in molecular motion of membrane with increase in incubation time. C) Low field EPR line showing the peak to peak intensity of unbound $\left(\mathrm{U}_{0}\right)$ and bound plus unbound (Bo+Uo) contributions. 
This is an author-produced, peer-reviewed version of this article. The final, definitive version of this document can be found online at Current Eye Research, published by Taylor \& Francis. Copyright restrictions may apply. doi: 10.1080/02713683.2020.1786131

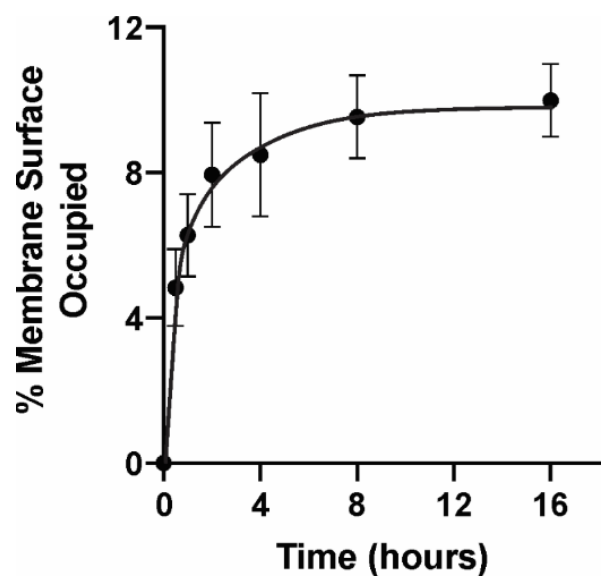

Figure 3. The membrane surface occupied by $\alpha$-crystallin increases with an increase in incubation time and is maximum about 8 hours. Error bars are estimated from the average of three independent experiments.

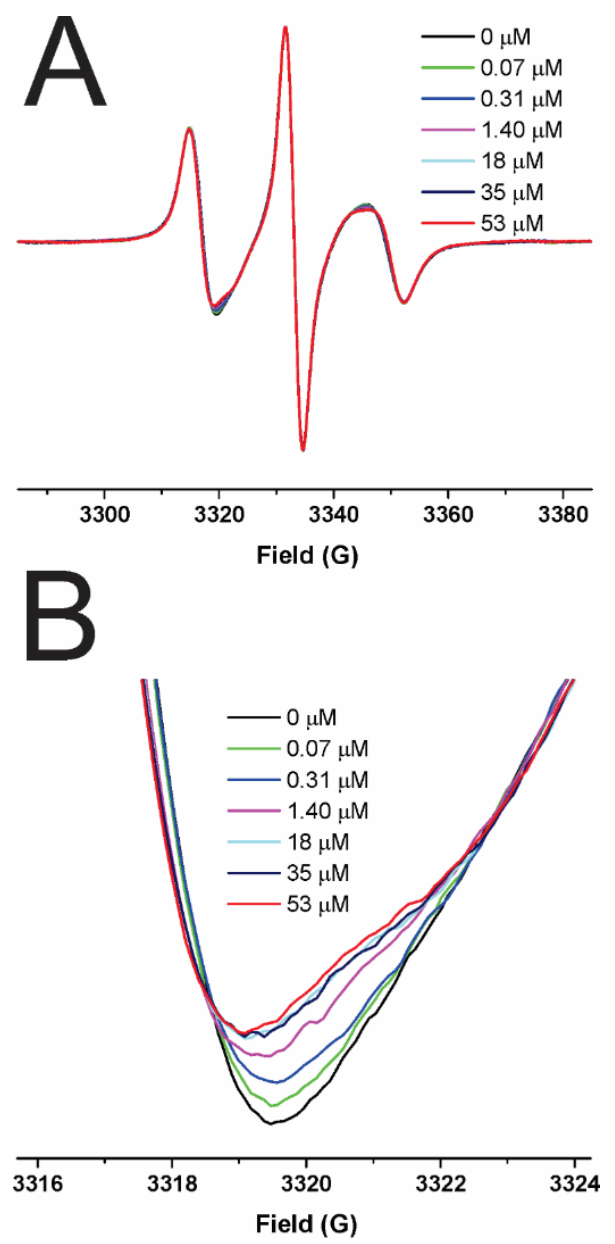

Figure 4. A) EPR spectra of CSL in membrane with varying concentration of $\alpha$-crystallin. The concentration of POPC was fixed at $9.4 \mathrm{mM}$. The mixture of $\alpha$-crystallin and membranes samples were incubated at $37{ }^{\circ} \mathrm{C}$ for $16 \mathrm{hrs}$. B) Fragment of EPR spectra demonstrating the change in molecular motion of membrane with an increase in $\alpha$-crystallin concentration. Membrane samples containing $0 \mu \mathrm{M}$ of $\alpha$-crystallin are used as control. 
This is an author-produced, peer-reviewed version of this article. The final, definitive version of this document can be found online at Current Eye Research, published by Taylor \& Francis. Copyright restrictions may apply. doi: 10.1080/02713683.2020.1786131

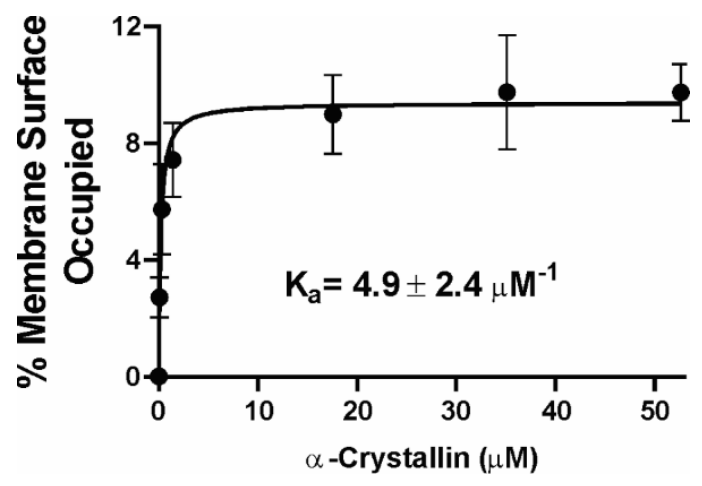

Figure 5. Binding of $\alpha$-crystallin with phospholipid membrane. The concentration of $\alpha$-crystallin was varied and POPC was fixed at $9.4 \mathrm{mM}$. The membrane surface occupied by $\alpha$-crystallin is estimated using equation 4 and Fig. 4. Error bars are estimated from the average of three independent experiments. Data points are fitted with one-site ligand binding model in GraphPad Prism (San Diego, CA) to estimate the binding affinity $\left(\mathrm{K}_{\mathrm{a}}\right)$.
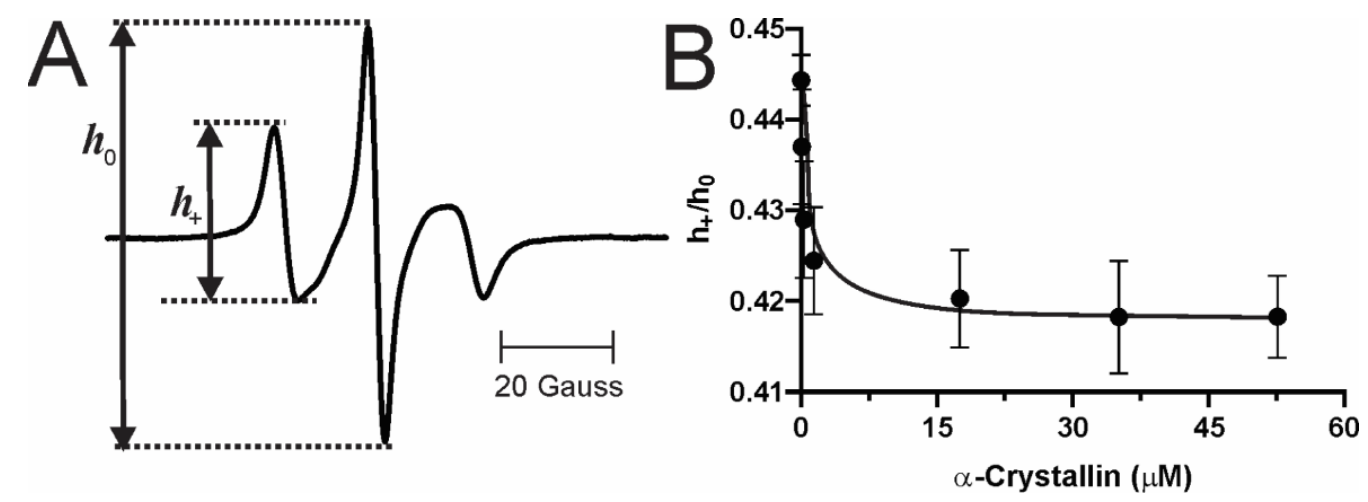

Figure 6. A) Representative EPR spectra of CSL from phospholipid membrane incubated with $\alpha$-crystallin (53 $\mu \mathrm{M}$ ) at $37{ }^{\circ} \mathrm{C}$ for $16 \mathrm{hrs}$. Spectra was recorded at $37^{\circ} \mathrm{C}$. Measured values used to calculate the mobility parameter are indicated. B) Mobility parameter $\left(h_{+} / h_{0}\right)$ obtained at $37^{\circ} \mathrm{C}$, is plotted as a function of $\alpha$-crystallin concentration. The POPC concentration was fixed to $9.4 \mathrm{mM}$. Error bars are estimated from the average of three independent experiments. As discussed in Sect. 2.1, there may be some contribution of the impurities in the change in membrane properties and this can be eliminated by using recombinant pure $\alpha$-crystallin. 
This is an author-produced, peer-reviewed version of this article. The final, definitive version of this document can be found online at Current Eye Research, published by Taylor \& Francis. Copyright restrictions may apply. doi: 10.1080/02713683.2020.1786131

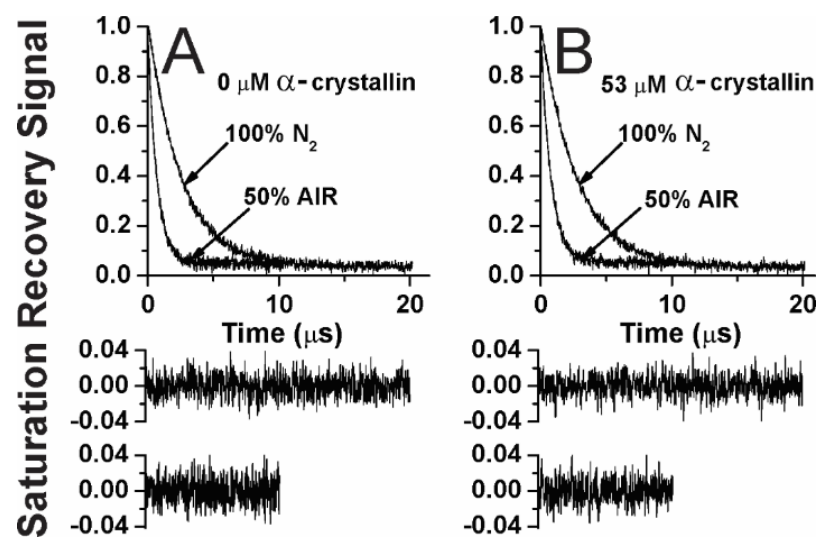

Figure 7. Representative SR signals with fitted curves and residuals (the experimental signal minus the fitted curve) for CSL in phospholipid membrane (concentration of POPC was fixed to $9.4 \mathrm{mM}$ ) incubated with $0 \mu \mathrm{M} \alpha$-crystallin (A) and with $53 \mu \mathrm{M} \alpha$-crystallin (B). Incubation was done at $37^{\circ} \mathrm{C}$ for $16 \mathrm{hrs}$. Signals were recorded at $37^{\circ} \mathrm{C}$ for samples equilibrated with $100 \%$ nitrogen, and with a gas mixture of $50 \%$ air and $50 \%$ nitrogen (A, B). SR signals were satisfactorily fitted to a single-exponential function both in the absence and presence of molecular oxygen with time constants of $2.52 \pm 0.01 \mu \mathrm{s}\left(\mathrm{A}, 100 \% \mathrm{~N}_{2}\right), 2.74 \pm 0.01 \mu \mathrm{s}\left(\mathrm{B}, 100 \% \mathrm{~N}_{2}\right), 0.68 \pm 0.004 \mu \mathrm{s}(\mathrm{A}, 50 \% \mathrm{AIR}), 0.78 \pm$ $0.004 \mu \mathrm{s}$ (B, $50 \%$ AIR), (both the upper and lower residuals are for a single-exponential fit).
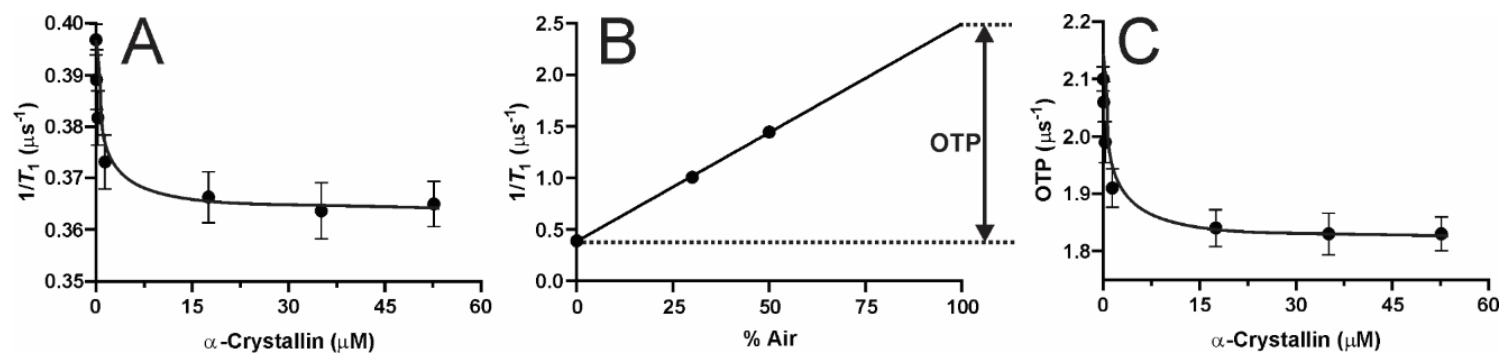

Figure 8. A) Profiles of the $T_{1}^{-1}$ (the spin-lattice relaxation rate) obtained at $37{ }^{\circ} \mathrm{C}$ with CSL spin label in the phospholipid membrane incubated with varying amount of $\alpha$-crystallin. B) $T_{1}^{-1}$ for CSL in phospholipid membrane incubated with $0 \mu \mathrm{M} \alpha$-crystallin plotted as \% air in the equilibrating gas mixture. The plot shows a linear dependence up to $50 \%$ air, and extrapolation of linear plot is performed to $100 \%$ air to indicate a way to calculate OTP. The same procedure is used to calculate OTP for phospholipid membrane incubated with varying amount of $\alpha$-crystallin C) Profiles of OTP obtained at $37^{\circ} \mathrm{C}$ with CSL spin label in the phospholipid membrane incubated with varying amount of $\alpha$-crystallin. Incubation was done at $37^{\circ} \mathrm{C}$ for $16 \mathrm{hrs}$ with POPC concentration fixed to $9.4 \mathrm{mM}$. Error bars are estimated from the average of three independent experiments. As discussed in Sect. 2.1, there may be some contribution of the impurities in the change in membrane properties and this can be eliminated by using recombinant pure $\alpha$-crystallin. 